sciendo
Management Consulting Journal

Volume 1.1 | June 2018

DOI: $10.2478 / \mathrm{mcj}-2018-0004$

ISSN: 2631-987X

\title{
Evolution or Revolution? Alternative Assignment Scopes
}

\author{
Brian Ing
}

\section{Introduction}

This is an exploration, based on similar concepts derived for the physical sciences by Thomas Kuhn, of the nature of change consultants propose for their clients. A distinction is made for assignments which are revolutionary and those which are evolutionary. It is noted that the latter strongly predominate and of themselves alone provide the benefits which justify the existence of our management consultancy profession. Finally, the position of a consultant proposing change which could be of either category to an organisation is discussed.

\section{'Change Agents'}

At one time it was quite popular to call management consultants 'change agents' (Tisdall, 1982). Perversely paradoxically at that time it was relatively rare for management consultants to be involved in implementing change, even advising on how to implement change. In some countries, the professional body expressed a strong opinion that implementation was not part of the professional scope of a management consultant. The term, 'change agent', faded from use, arguably through its association through the word 'agent' implying action and getting things done. However, for our purpose today the alternative title for what we do stresses that a high proportion of our professional efforts are involved with change. Of course, more recently consulting on implementation (perhaps given a different nomenclature such as 'strategy deployment') and actually being involved with implementation of change (and we now have many practices specialising in change management) are part of the mainstream of consulting practice. But the basis of all work is change in all its forms, expected, to be defended against and perceived.

We have a whole taxonomy of types of change. Be it strategic or tactical. Be it radical or piecemeal. Both of which are binary choices. Others are a more scaled variable, such as changes affecting small proportions of an organisation in gradual steps to a change affecting the entire organisation.

This conceptual paper concerns a one such binary descriptor and its use in a consulting practice: evolution or revolution. 


\section{Thinking from Elsewhere}

As a young profession, we have been profligate resourceful in acquiring as our base knowledge, the knowledge, insights, and techniques from many other professions. The obvious ones were production management, finance, marketing, and information technology, which clearly related to the business fundamentals of the organisations which were our clients. But a much wider range have fed our base, such as psychology, sociology, and anthropology. The competent management consultant will have a collection of tools and knowledge from a wide range of other professions.

This article is based on applying some understandings derived for the hard sciences by Thomas Kuhn in his book 'The Structure of Scientific Revolutions' (1962). To simplify his now widely accepted thesis is that most science practised by its professionals is 'normal' science. That is to say, the situations being addressed with tools and accepted theories and laws. The very high proportion of all science work is of this form. However, using accepted wisdom and applying to whole range of phenomena and situations will occasionally reveal where the application of accepted ideas does not result in agreement with what happens in nature. Initially, these unexpected circumstances are treated as oddities, often with the expectation of an improved use of a wider range of accepted knowledge will resolve the apparent puzzle. As more and more of these unexplained phenomena are found, the science community becomes more unsettled and is ready to consider that maybe that all about the situation is not known. Suddenly, one person (or a small group) comes forward, almost bursts through, with a totally different explanation (sometimes requiring the questioning or replacement of what had until then been accepted wisdom). Thomas Kuhn coined the phrase 'paradigm shift' to describe this fundamental move in thinking (Kuhn, 1962).

The classic example in physics is Einstein's Theory of Relativity replacing the twohundred-year-old Newtonian position on relativity. In the world of physics this was such a fundamental change that we still talk of it today over a hundred years later. At the same time, quantum theory was born. Modern Physics became the accepted approach instead of 'Classical Physics' and the application of these new ideas can be said to have defined the last century's progress. Truly justifying the term Kuhn applied - 'revolution'.

\section{Evolution and Revolution}

In scientific disciplines these revolutions happen quite rarely (although in the biological sciences, it seems the gap between revolutions is shrinking). So, are such dramatic revolutions in thinking and practice also so rare in the fields of the clients of management consultants? There are only a relatively small number of sciences, and they are global. Business and organisations cover different sectors, different marketplaces, different cultures and economies. We show later some recent examples of revolutions in our world. But these are still the minority of assignments, while normal accepted practice is applied over and over again to achieve incremental improvement in performance (where that is much wider than just financial performance) for organisations. This type of assignment will be referred to as 'evolution' taking as it does the original organisation and amending it in selected ways to achieve the improvement in performance.

The remainder of this article will use the evolution or revolution descriptor of change and discuss the implications for practising management consultants. 


\section{Revolution Examples}

A classic revolutionary change assignment started with the question 'what business are we in?' (Levitt, 1960). Examples are legion of the result of answering this question has changed the focus of attention from what the organisation does (eg. fly aeroplanes) to definitions of what the customers obtain from dealing with the company (eg. able to get to different places to do business, learning, or tourism). This apparently simple change of central intent affects the organisation throughout in fundamental ways. The initial use of this question was a revolution in consulting practice.

'Get the customer to do the work' and other advantages from getting customers, clients and those interacting with the organisation through using the internet, speeding response times, reducing costs and, with good design, improving satisfaction are now so commonplace. But for the author of this article, it was regarded as a sackable offence for predicting for customers of banks in the 1970s they would want to electronically query their data held by the bank. This was after hearing what had been achieved with ARPAnet. This research network was improved and renamed Internet.

There are many other examples. Some are innovations in technology having a wide and dramatic impact (see for instance the provision of VisiCalc, an early spreadsheet program, on an Apple Ile) others are changes in cost points that dramatically enable changes in business practices (qv. the PC, the photocopier, and the mobile phone). These examples do not arise within the management consultancy profession. However, as happens often, it is our profession that learns from early adoptions of these new approaches the firms consolidate experience into best practice and guidance for others to follow.

The revolution in business practice may be led by a concept, an idea or even a mantra (even if delivered via hardware and software). For example, just in time supply chains; quality management; 'client-server' computing; cloud-based computing; and 'stick to your knitting'. More recently, we have become familiar with the phrase 'disruptive change'. Totally new ways of satisfying customer requirements are achieved through the use of emerging technology. Who would not describe the impact Amazon has had, initially on book selling but now all aspects of retail, as anything else but 'revolutionary'? It is interesting to note that often these disruptive revolutionary changes are achieved by new organisations, not by those organisations currently serving the customers.

Perversely, these disruptive changes in the business environment require management consultants to use their normal approach to new ideas. That is to understand the matter, its impacts on businesses, the responses they should make and the ways of achieving those changes. We need to review what others have done and learn and consolidate all into our advice to clients. Something we have been doing for every change in doing business since we were organised as a profession.

"There have been many examples of revolutions in the overall business, including the third sector, world. But no matter how many there have been, the number of assignments performed by management consultants that can be considered revolutionary are relatively very small compared to the vast majority of assignments which can be classed as evolutionary."

Often, revolutionary new approaches are developed by a small group of individuals. They may be academics, business commentators or even a consultant with an idea. Once the idea is first defined and has limited exposure outside the original experimentalists, it is 
often that niche boutique consultancies work with early adopter clients to refine and confirm the approach. Once some success is achieved, it is common for the large practices, who are always seeking to be able to introduce new ideas to their clients and have the resources to be able to service quickly a wide range of clients. At the same time business schools take notice and start research. A couple of years later, the topic is introduced into the courses, especially the MBA, and gradually managers in business become aware and later proficient in the use of the approach. The sales of the approach by the large consultancies decline from their peak, but at the same time consultancies specialising in, and expert in, the new approach start selling to the managers now fully aware if not competent in the new approach. Latterly, consulting services are still sold where the business does not have sufficient resources although the managers are now fully trained and competent in the approach.

Thus the new approach has, like any service product, a product life cycle. It is only at the very early stages that the approach can be usually described as revolutionary. Soon, typically in the early period of adoption by the larger consultancies, the approach is properly defined as accepted business best practice and the assignments are classified as evolutionary.

\section{Evolution Examples}

Almost all assignments use accepted knowledge and practice to advise and implement required change in clients. Some might still have a dramatic change on the client organisation. Often a marketing strategy assignment can lead to completely different futures for a client and its staff. But the basis of the suggested change is still accepted practice: just that this has been applied to the situation of the client.

Business process improvements, cost engineering, staffing number reviews are all examples of evolution projects. The organisation, or part of it, is evolved from its current state to an improved one. It is the stock in trade of most consultants. The assignments can still be exemplary, the client interaction and the overall assignment can be superb, even prize winning in national and international competitions. This is the bulk of the benefits brought to the organisations across the world that justifies the investment in using consultants. Indeed, the cumulative benefits more than justify the existence and practice of management consultants. The assignments might not be as exciting, but they are valuable and valued.

Without a single revolutionary assignment, our profession is justified by the benefits derived by these assignments. The average multiplier of the consultancy fee to the benefits obtained, has been estimated many times but no study has found the costs to exceed the benefits. This is a replica of the conclusions reached by Thomas Kuhn. 'Normal' science had and continues to produce economic value far in excess of the total costs of the scientists working with accepted practice and theory. But...

\section{The Client View}

All the above is predicated on the view of the consultant. Revolutions, in the sense of ground-breaking paradigm shifts, come rarely. Once they have been understood, they soon become part of our toolbox for what we see as evolutionary change. 
But clients can, but not always, become so focused on their organisations that they are unaware of some new thinking. They can also be so involved with the logic, rationale, history and culture of their organisation that their resistance to change is heightened. Working closely with colleagues within the organisation can produce a 'group-think' (Whyte, 1952) that what they do is the right way to proceed. A consultant proposing a change based on the accepted norms can be reviewed as proposing an unexpected radical change. Or to use our terminology, a revolution.

Therein lies the potential for a delightful irony. The consultant sees the change as complying with known norms and expectations, and the client sees it as a fundamental change to an organisation not yet prepared for so widespread or fundamental change. This can influence the consultant's communication with the client during the process of gaining the assignment. Bearing in mind that the client view is based on their perception, it will be for them a true revolutionary change.

\section{Which Assignment to Do?}

Marketing people distinguish between 'need' and 'want' for deciding marketing approaches. Until a need becomes a want, there is little point in trying to market or sell the product or service. But with consultancy a third consideration arises. What type of assignment will I be able to convince the client to buy? The process from initial engagement, investigation, and exploration of the requirements for consultancy, there is often an extensive dialogue and interaction with the client. Some say, there is often more consulting depth in the sales process for an assignment than in performing the resultant assignment.

An example of a dilemma posed in the past when BPR was fashionable will suffice to illustrate the choices for the consultant. An organisation may be considerably underperforming its competition, or in danger of becoming so. The need is probably for a radical change. The person interacting as the lead client contact with the consultant may have different views on the level of change necessary from others on the board. They may be aware of the growing reputation for BPR implementations causing job losses, while the vested interests of the individual client may be a factor, the risks associated with revolutionary assignments is higher than evolutionary one; but the benefit is potentially much higher too.

In essence, the consultant needs to consider the needs and wants of the organisation and the process of the procurement of the consultancy in order to decide what should be offered. In the worst case, the consultant will decide that what is required is not going to be procured. This may cause the ethical consultant to withdraw (see ICMCI Code of Professional Conduct). Being able, as a consultant, to make the necessary choices and prepare the client for the suggested assignment proposal is a key consultancy competence.

Those interested in overall consulting competences should see the ICMCI Competence Framework: the requirements of consultants interacting with clients in the provision of a management consultancy service have been recently defined in the ground breaking new ISO standard, ISO20700 (obtainable from ISO and all national Standards Bodies) which is the first 'service' standard to written for any profession or service provider. The considerations for a competent professional management consultant in deciding which service to offer are extensive and complex and would be the basis for many future articles. 


\section{References}

Agents of Change: Development and Practice of Management Consultancy, Patricia Tisdall Nov 1982, Institute of Management Consultants.

As a number of professionals identifying as such from the early years after WWII. Individuals much earlier have since been described as management consultants although they would not have recognised the term (e.g. Samuel pepys in his diaries 1960 to 1969)

Kuhn, Thomas S. (1962). The Structure of Scientific Revolutions (1st ed.). Chicago, IL: University of Chicago Press. ISBN 9780226458113

Levitt, T. (1960). "Marketing Myopia". Harvard Business Review.

Michael Hammer, "Reengineering Work: Don' t Automate, Obliterate", Hardvard Business Review, July, 1990 and subsequent writings

Groupthink" by William H. Whyte Jr. in Fortune magazine, March 1952

See the ICMCl standard in www.cmc-global.org/content/cmc-standards, Appendix 1 